Laory, I., Bel Hadj Ali, N., Trinh, T., and Smith, I. "Measurement System Configuration for Damage Identification of Continuously Monitored Structures." J of Bridge Engineering, Vol. 17, 2012, SPECIAL ISSUE: Nondestructive Evaluation and Testing for Bridge Inspection and Evaluation, pp 857-866 http://cedb.asce.org Copyright ASCE

\title{
Measurement system configuration for damage identification of continuously monitored structures
}

\author{
Irwanda Laory ${ }^{\mathrm{a}}$, Nizar Bel Hadj Ali ${ }^{\mathrm{b}}$, Thanh N. Trinh ${ }^{\mathrm{a}}$, Ian F. C. Smith \\ ${ }^{a}$ Swiss Federal Institute of Technology Lausanne (EPFL), Station 18, CH-1015 Lausanne, Switzerland \\ ${ }^{b}$ Ecole Polytechnique de Tunisie, University of Carthage, B.P. 743, La Marsa 2078, Tunisia
}

1

\section{Abstract}

3

Measurement system configuration is an important task in structural health monitoring in that decisions influence the performance of monitoring systems. This task is generally performed using only engineering judgment and experience. Such approach may result in either a large amount of redundant data and high data-interpretation costs, or insufficient data leading to ambiguous interpretations. This paper presents a systematic approach to configure measurement systems where static measurement data are interpreted for damage detection using model-free (non-physicsbased) methods. The proposed approach provides decision support for two tasks: (1) determining the appropriate number of sensors to be employed and (2) placing the sensors at the most informative locations. The first task involves evaluating the performance of measurement systems in terms of the number of sensors. Using a given number of sensors, the second task involves configuring a measurement system by identifying the most informative sensor locations. The locations are identified based on three criteria: the number of non-detectable damage scenarios, the average time to detection and the damage detectability. A multi-objective optimization is thus carried out leading to a set of non-dominated solutions. To select the best compromise solution in this set, two multi criteria decision making methods, Pareto-Edgeworth-Grierson multi-criteria decision making (PEG-MCDM) and Preference Ranking Organization METhod for Enrichment Evaluation (PROMETHEE), are employed. A railway truss bridge in Zangenberg (Germany) is used as a case study to illustrate the applicability of the proposed approach. Measurement systems are 
configured for situations where measurement data are interpreted using two model-free methods:

Moving Principal Component Analysis (MPCA) and Robust Regression Analysis (RRA). Results demonstrate that the proposed approach is able to provide engineers with decision support for configuring measurement systems based on the data-interpretation methods used for damage detection. The approach is also able to accommodate the simultaneous use of several model-free data-interpretation methods. It is also concluded that the number of non-detectable scenarios, the average time to detection and the damage detectability are useful metrics for evaluating the performance of measurement systems when data are interpreted using model-free methods.

Subject headings: Bridges; damage; monitoring; measurement; optimization; decision making

Keywords: Measurement system configuration; model-free data interpretation; damage detectability; multi-objective optimization; multi-criteria decision-making

\section{Introduction}

Recent advances in sensor technology and data acquisition systems enable engineers to continuously monitor civil engineering infrastructures so that damage can be detected before it reaches a critical level. Many structures have been monitored using sophisticated measurement systems with a large number of sensors. The cable-stayed Stonecutters Bridge in Hong Kong, for example, is equipped with more than 1200 sensors, including accelerometers, temperature sensors, strain gauges and other sensors ( $\mathrm{Ni}$ et al. 2008). In many cases, due to the lack of systematic approaches for the configuration of measurement systems, the number of sensors and their locations were determined using engineering judgement alone. This approach may result either in a large amount of redundant data or insufficient data. Redundant data leads to high data-interpretation costs while insufficient data results in ambiguous interpretations. Therefore, a systematic approach for measurement system configurations that provide good performance for damage identification is desirable.

Damage in civil structures may be identified by interpreting monitoring data collected using measurement systems. The task of measurement interpretation falls into the broad area of system 
identification. There are generally two classes of data-interpretation methods in system identification: model-based (physics-based) methods and model-free (non-physics-based) methods (ASCE 2011). Model-based data-interpretation methods typically utilize measurement data to identify models that are able to predict the real behaviour of structures (Aref et al. 2005; Chen and Wu 2010; Jaishi and Ren 2006; Koh and Thanh 2009; Robert-Nicoud et al. 2005a). However, for civil infrastructure, creating such models is often difficult, expensive and may not reflect the real structural behaviour because of the uncertainties that are common in complex civil-engineering structures (Goulet et al. 2010). Furthermore, model-based methods are not necessarily successful in identifying structural behavior (Saitta et al. 2005).

Model-free data-interpretation methods, on the other hand, involve interpreting measurement data without geometrical and material information (i.e. without structural models). These methods identify damage by tracking changes in time-series signals; thus, they are well-suited for interpreting measurements during continuous monitoring of structures. Liu et al. (2009) developed a limit state equation for safety evaluation of existing bridges. Hou et al. (2000) proposed a wavelet-based approach for structural damage detection. Omenzetter and Brownjohn (2006) proposed an autoregressive integrated moving average model method for damage detection. Lanata and Grosso (2006) applied a proper orthogonal decomposition method for continuous static monitoring of structures. Yan et al. (2005) proposed local PCA-based damage detection for vibration-based structural health monitoring. Posenato et al. $(2010 ; 2008)$ proposed two model-free datainterpretation methods: (1) Moving Principal Component Analysis (MPCA) and (2) Robust Regression Analysis (RRA), to detect and localize damage in civil engineering structures. These two methods were compared with many other methods and it was demonstrated that their performance for damage detection was superior to the other methods when dealing with civil-engineering challenges that include high noise, missing data and outliers.

Configuration of measurement systems is based on the methods that are used to interpret measurement data (i.e. different methods may result in different measurement configurations). 
Thus, in order to maximize the performance of measurement systems, data-interpretation methods should be selected prior to configuration task. Previous studies (Kang et al. 2008; Li et al. 2007; Li et al. 2004; Liu et al. 2008; Meo and Zumpano 2005; Papadimitriou 2004) have mainly focused on the configuration of measurement systems for dynamic tests where measurement data is interpreted using model-based methods. Li et al. (2007) investigated and compared two measurement system configuration methods, modal kinetic energy and effective independence, for damage identification using dynamic tests. Kang et al. (2008) proposed a virus co-evolutionary partheno-genetic algorithm, which combined a partheno-genetic algorithm with virus evolutionary theory, to place sensors on a large space structure for the purpose of modal identification. Meo and Zumpano (2005) investigated six different measurement system configuration techniques for optimum identification of structural vibration characteristics. For multiple-model methods using static measurements, Robert-Nicoud et al. (2005b) proposed an iterative greedy algorithm to design a measurement system that gives maximum separation between predictions of candidate models. Kripakaran and Smith (2009) utilized damage scenario generation and proposed strategies for two measurement tasks: (1) configuring initial measurement systems and (2) enhancing these systems for subsequent measurements once data interpretation is carried out. Few studies have used damage scenario generation as a starting point for measurement system configuration. Although many studies have been performed to design measurement systems for structural identification, none have studied the measurement system configuration for model-free data-interpretation methods using static measurements.

The number of potential configurations for a measurement system is exponentially related to the number of possible sensor locations (Saitta et al. 2006). Hence, the task of configuring measurement systems is best carried out using global search algorithms. In several studies (Kripakaran and Smith 2009; Liu et al. 2008; Rakesh and et al. 2008; Rao and Ganesh 2007; Tongpadungrod et al. 2003; Wang et al. 2002; Xu et al. 2010), stochastic search techniques were employed for measurement system configuration. The evaluation of the potential configurations should include several criteria (objectives). For example, good configurations have a minimum number of sensors with a maximum 
performance. For optimizing placements of active control devices and sensors, Cha et al. (2011) proposed a methodology that minimizes the number of employed devices and sensors while maximizing structural performance under earthquake. In most cases, objectives are noncommensurable (i.e. they are measured in different units) and usually in conflict with each other. There may be no solution satisfying all objectives simultaneously. Thus, the solution is often a set of non-dominated solutions (Pareto-optimal solutions), or a compromise solution according to engineers' preferences.

The task of selecting a compromise solution falls into the field of multi-criteria-decision-making (MCDM). Grierson (2008) proposed Pareto-Edgeworth-Grierson multi-criteria decision-making (PEGMCDM) that employs a trade-off-analysis technique to identify compromise solutions for which the competing criteria are mutually satisfied in a Pareto-optimal sense. The PEG-MCDM procedure can be effectively applied to MCDM tasks that involve many objectives and feasible solutions. Another method for MCDM is Preference Ranking Organization METHod for Enrichment Evaluation (PROMETHEE) (Behzadian et al. 2010; Brans and Mareschal 2005a; Brans 1982; Brans et al. 1986). This method utilizes a preference index to compute a net flow for each Pareto optimal solution. This value is then used to rank the Pareto optimal set. Bel Hadj Ali and Smith (2010) compared PEGMCDM and PROMETHEE for vibration control of a tensegrity structure.

This paper presents a systematic method-based approach to configure measurement systems where static measurement data are interpreted using model-free (non-physic-based) methods. The approach involves damage scenario generation, optimization of several criteria and multi-criteria decision-making. It consists of two steps. The first step is to provide decision support for engineers to determine the number of sensors to be employed. The second step is to configure sensor locations based on three criteria: the number of non-detectable scenarios, damage detectability and the average time to detection. A genetic algorithm (Sastry 2007) is employed to evaluate potential configurations based on a multi-objective optimization. Then, two multi-criteria decision-making methods, PEG-MCDM and PROMETHEE, are applied to provide support for identifying the best 
compromise configuration. To illustrate the performance of the proposed approach, measurement systems are configured for the Zangenberg railway bridge in Germany, where the measurement data are interpreted using Moving Principal Component Analysis and Robust Regression Analysis.

\section{Model-free (non-physics-based) data-interpretation methods}

\section{Moving principal component analysis (MPCA)}

MPCA is a modified version of principal component analysis (PCA) (Hubert et al. 2005). PCA is a mathematical process of transforming a number of possibly correlated variables into a smaller number of uncorrelated variables, called principal components. The first few components retain most of the variation present in the original variables. In the context of structural health monitoring, PCA is employed to enhance the discrimination between features of undamaged and damaged structures and to reduce computational time. Posenato et al. (2008) proposed "moving" PCA (MPCA) that essentially applies PCA to a moving constant-sized window of measurements instead of the whole dataset. MPCA is applied to measurement time histories by first constructing a matrix that contains the history of all the measured parameters and second iteratively extracting datasets corresponding to a moving window and computing the principal components using PCA.

The principal components are the eigenvectors of the covariance matrix of extracted measurements. Sorting the eigenvectors by eigenvalues in decreasing order, the components are arranged in order of significance. The first few principal components contain most of the variance of the time series while the remaining components are defined by measurement noise. Thus, MPCA is carried out by analyzing only the eigenvectors that are related to the first few eigenvalues. When damage occurs, mean values and components of the covariance matrix change and as consequence, so do values of eigenvalues and eigenvectors. An advantage of using a moving window rather than whole measurements is less computational time and earlier damage detection since very old measurements do not bias results. Another advantage is adaptability. Once new behaviour is identified, adaptation enables detection of further damage. 
Robust regression analysis (Andersen 2008; Jajo 2005) involves assigning a weight to each data point using a process called iteratively reweighted least squares. This method achieves more reliable results than linear regression analysis when measurement data are subjected to outliers. RRA is applied for continuous monitoring of structures by finding all sensor pairs that have a high correlation and then to focus on the correlation of these couples to detect anomalies. To find sensor pairs with a high correlation, the correlation coefficients $r_{s i, s j}$ between measurements from two sensors $s_{i}$ and $s_{j}$ are computed and compared with the correlation coefficient threshold that is chosen to be 0.8 in this study. All sensor pairs having a correlation coefficient greater than the threshold are selected in order to formulate the robust regression model. The linear relation between $s_{i}$ and $s_{j}$ is written as

where $s_{j}^{\prime}$ represents the value of $s_{j}$ computed according to the linear relation. $a$ and $b$ are the coefficients of the robust regression line estimated from measurements. These coefficients are estimated using iteratively reweighted least squares. The robust regression analysis is carried out by observing the difference between the measurements $s_{j}$ and the prediction by linear regression-line $s^{\prime}{ }_{j}$, called regression residuals. Standard deviation of the residuals is used to define the threshold of confidence intervals for each pair. Damage is identified when the value exceeds the confidence interval. In addition to the advantage of being insensitive to outliers and missing data, RRA is capable of adapting to the new state of a structure and thus permitting the identification of further anomalies.

\section{Task formulation and optimization}

172 Measurement system configuration involves placing sensors at the most informative locations such that the performance of damage detection is maximized. The number of sensors to be placed and 
potential sensor locations leads to a space of possible measurement system configurations. Even with a small number of possible sensor locations, it is practically impossible to generate and test all configurations due to the combinatorial nature of the task. Deterministic optimization methods, for example "branch and bound" may be able to treat small combinatorial tasks. However, evaluating all combinations of $i$ sensors among $n$ possible locations has the following computational complexity:

$$
\sum_{i=1}^{n} C_{n}^{i}=2^{n}-1
$$

Stochastic search is particularly useful in such situation. Stochastic methods support search well in complex and large solution spaces. Although there is no guarantee of reaching a global optimum, near optimal solutions are usually obtained.

In this paper, three objective functions are used to evaluate a configuration of sensor placements represented by a vector of $\mathrm{N}$ decision variables $\mathbf{x}^{t}=\left[x_{1}, x_{2} \ldots, x_{N}\right]$. The first objective function $f_{1}$ is to evaluate the number of non-detectable damage scenarios for a measurement configuration. The second objective function $f_{2}$ is to evaluate the damage detectability which is defined through the average of the minimum detectable damage level using MPCA and RRA as follows:

$$
\text { Damage detectability }(\%)=100 \% \text { - Minimum detectable damage level (\%) }
$$

where the minimum detectable damage level is the smallest percentage loss of member-stiffness that can be detected. The third objective function $f_{3}$ is to evaluate the average time-to-detection associated with a measurement configuration. Time-to-detection is the period (in days) from the moment damage occurs in the structure to the moment damage is detected. The value of $f_{3}$ is obtained by averaging the time-to-detection for the whole set of detected damage scenarios.

From these three objective functions, measurement system configuration is formulated as a multiobjective optimization task that results in a set of possible solutions. Solutions are known as Pareto optimal (non-dominated) solutions. In a multi-objective minimization task, a solution is called Pareto 
optimal if there is no other solution that satisfies one objective function more without having a worse value for at least one other objective function. Many evolutionary multi-objective optimization methods have been used in various fields due to their effectiveness and robustness in searching for a set of trade-off solutions (Coello Coello et al. 2007). In this study, measurement system configurations are represented by a finite number of discrete variables. Two stochastic search algorithms are tested for this task: Probabilistic Global Search Lausanne (PGSL) (Raphael and Smith 2003) and Genetic Algorithms (GA) (Sastry 2007). The genetic algorithm is finally adopted for the multi-objective optimization task where optimization variables are coded as integer strings.

\section{Multi-criteria decision making}

In order to identify a good solution among the set of the Pareto optimum solutions for configuring a measurement system, our approach employs two Multi-Criteria Decision Making (MCDM) methods: Preference Ranking Organization METHod for Enrichment Evaluation (PROMETHEE) (Brans and Mareschal 2005a; Brans 1982; Brans et al. 1986) and Pareto-Edgeworth-Grierson multi-criteria decision making (PEG-MCDM) (Grierson 2008).

\section{Preference Ranking Organization METHod for Enrichment Evaluation (PROMETHEE)}

The PROMETHEE method was developed as a MCDM method to solve discrete decision tasks with conflicting criteria to establish ranking of Pareto-optimal solutions with conflicting criteria. Incorporating preferences is also considered to help to handle conflicting objectives (Fleming et al. 2005). An aggregated preference index is used to compute outranking flows for each Pareto optimal solution. These outranking flows are then exploited to establish a partial ranking (PROMETHEE I) or a complete ranking (PROMETHEE II) on the Pareto set.

Let $s_{1}, \ldots, s_{n}$ be $n$ Pareto optimal solutions and $f_{1, \ldots,} f_{m}$ denote the $\mathrm{m}$ decision criteria for PROMETHEE I., The PROMETHTEE procedure is based on pairwise comparisons between Pareto optimal solutions. This method assumes that the preference between two solutions for a given criterion can be expressed using ratios. Brans and Mareschal (2005b) proposed six types of preference functions 
223 the criterion $k$ by a real value in the interval $[0,1]$. Using preference functions associated with all decision criteria, an aggregate preference index $C\left(S_{i}, S_{j}\right)$ is thus defined in Eq.(4), where $w_{k}$ are

225 weights expressing the relative preference of the decision criteria.

Once the aggregate preference indexes are computed for each pair of the Pareto solutions, outranking flows can be evaluated. The preference flows $\left(\varphi^{+}, \varphi^{-}\right.$and $\left.\varphi\right)$ for each solution are formulated as follows:

$$
\varphi^{+}\left(S_{i}\right)=\sum_{j=1}^{n} C\left(S_{i}, S_{j}\right)
$$

solutions in the solution set. The negative flow $\left(\varphi^{-}\left(S_{i}\right)\right)$ expresses the intensity of preference of all other solutions over solution $S_{i}$. The difference between the positive and the negative flow gives the net preference flow $\left(\varphi\left(S_{i}\right)\right)$, which is the absolute preference of the solution $S_{i}$ over all other solutions in the solution set. For PROMETHEE II, this value is used to establish a complete ranking of all Pareto optimal solutions.

\section{Pareto-Edgeworth-Grierson multi-criteria decision making (PEG-MCDM)}

Grierson (2008) proposed a MCDM strategy employing a trade-off-analysis technique to identify

compromise solutions for which the competing criteria are mutually satisfied in a Pareto optimal set.

242 Grierson (2008) formulated the PEG-theorem which states existence and uniqueness of a Pareto-

243 compromise solution that represents a mutually agreeable trade-off between conflicting criteria for 
multi-objective optimization tasks. The PEG-MCDM method is summarized here for the case of a two-criteria decision task. Refer to (Grierson 2008) for detailed description of a general case.

Having the Pareto-optimal set of solutions from a multi-objective optimization, let $f_{1}, \ldots, f_{n}$ denote the $n$ vectors that define the Pareto-optimal data constituted by $m$ Pareto-optimal solutions. The original Pareto data are first normalized to find m-dimensional vectors $\mathbf{x}_{i}$.

$x_{i}=f_{i}-f_{i}^{\min } / f_{i}^{\max }-f_{i}^{\min } ; \quad(i=1, n)$

The $m$ entries of each of the $n$ vectors are sequentially reordered from their minimum to maximum. For $n=2$ decision criteria, the Pareto data are thus represented by two $\mathrm{m}$-dimensional normalized vectors.

$\mathbf{x}_{1}^{T}=\left[x_{1}^{\min }, \ldots, x_{1}^{\max }\right]$ and $\mathbf{x}_{2}^{T}=\left[x_{2}^{\max }, \ldots, x_{2}^{\min }\right]$

In order to obtain a competitive equilibrium state at which a Pareto trade-off can take place between the two criteria, Grierson (2008) proposed an approach for transforming the Pareto data without changing its ordinal character so that a unique Pareto trade-off between two criteria is mutually agreeable. This is done by transforming the normalized Pareto curve to a circular Pareto that has only one competitive equilibrium state. In order to perform this transformation analytically, the criteria vectors $\mathbf{x}_{1}$ and $\mathbf{x}_{2}$ are uniformly shifted and then re-normalized to obtain vectors $\mathbf{x}_{1}^{*}$ and $\mathbf{x}_{2}^{*}$

$$
\mathbf{x}_{i}^{*}=\left(\mathbf{x}_{i}-\delta \mathbf{x}_{i}\right) /\left(1-\delta x_{i}\right) \quad(i=1,2) \quad ; \quad \delta x_{1}=\delta x_{2}=\sqrt{2}-1
$$

The objective criteria values corresponding to the unique competitive equilibrium point are evaluated.

$f_{i}^{0}=f_{i}^{\max }-\left(f_{i}^{\max }-f_{i}^{\min }\right)\left(\Delta r_{0}+\sqrt{2} / 2\right) ; \quad(i=1,2)$

where $f_{i}^{0}$ is the value of the two objective functions for the Pareto-compromise solution. $\Delta r_{0}$ is the radial shift from the transformed Pareto curve to the unique competitive equilibrium point. A ranking of the original Pareto data set may be achieved by computing the distance of the Pareto solutions to 
the Pareto-compromise solution. This distance is represented by the mean-square-error between the each of the $m$ original Pareto solutions.

\section{0}

271

\section{Measurement system configuration}

The aim of measurement system configuration is to enhance the effectiveness of data-interpretation tasks for monitoring of structures. Therefore, the performance of a measurement configuration is evaluated based on criteria associated with damage-detection capacity of data-interpretation methods. The proposed approach involves damage scenario generation, multi-objective optimization and multi-criteria decision-making. Damage scenarios depend upon structural factors such as material, geometry, structural characteristics and geographical location. These scenarios can be represented by the value of structural parameters which are specified by engineer. For example, damage in a structural element may be modelled as the percentage reduction in axial or flexural stiffness. Damage scenarios are employed as benchmark situations to evaluate the performance of a given measurement system.

As described in the task formulation section (section 3), measurement configuration involves multiobjective optimization task considering several criteria. Multi-objective optimization can lead to solutions with the minimal number of sensors and optimal placements in one step. However, in practical situations, measurement system configuration is often a weakly defined task where there are criteria that are not explicitly taken into account. Such criteria may include access for installation, additional measurement needs and sensor maintenance cost. In such situations, support tools that enable decision makers to be involved in the process are preferable. Therefore, instead of providing decision makers with optimal solutions according to incomplete criteria, explicit trade-off information is provided for the number of sensors versus performance in the first step. 
In order to obtain information about the trade-off, multi-objective optimization and multi-criteria

291 decision-making need to be performed iteratively for increasing number of sensors. The computational complexity (using $O$ notation) of such a task is as follows

where $C_{\text {total }}$ is the total complexity of the task, $C_{1}$ is the complexity of data-interpretation method,

$n_{1}$ is the number of damage scenarios, $n_{2}$ is the number of evaluations that is required to converge number of damage scenarios and evaluations are linearly proportional to the total complexity of the task. The number of damage scenarios and evaluations that are required for multi-objective optimization are higher than that for single-objective optimization. Multi-objective optimization can be carried out by transforming additional criteria into constraints in a single-objective optimization. The number of evaluations for such approach $\left(n_{\text {total }}\right)$ is the upper bound of the number of evaluations in multi-objective optimization as follows

where $n_{s}$ is the number of evaluations for a single-objective optimization, $P$ is the number of Pareto-points corresponding to an additional objective and $m$ is the number of objectives. Eq.12 shows that the total number of evaluations is exponentially related to the number of objectives. For example, assuming $n_{s}=400$ and $P=10$, the number of evaluations increases from 400 to 40000 when the number of objectives increases from 1 to 3 . The time required for an evaluation depends on factors such as algorithms that are used for data interpretation, the size of data and the computer system that is used to perform the task. For the situation that is studied in this paper, the computational time for one evaluation took about 5 seconds. Table 1 shows the results of the execution-time estimations for this example where the time to perform one evaluation is assumed to 
be 5 seconds. In comparison with single-objective optimization, performing multi-objective optimization leads to much higher computational costs. Furthermore, when the solution space becomes too large, performing multi-objective optimization is no-longer likely to obtain near optimal solutions.

Considering that not all information can be accounted for explicitly as well as the computational complexity associated with increasing number of objectives, measurement system configuration is carried out in two steps.

- A preliminary step using single-objective optimization to explore solution space in order to

The two-step procedure for measurement system configuration is illustrated in Figure 1 . In the first step, the solution space is explored by minimizing the number of non-detectable damage scenarios and by observing the improvement of the measurement system performance with respect to the increasing number of sensors. Engineers are thus able to determine the appropriate number of sensors through identifying where the addition of sensors will not give a significant improvement in performance.

Given the number of sensors to be employed, the second step is to configure measurement systems by identifying the best sensor locations. After the first step of preliminary exploration, this step conducts an in-depth exploration in a narrower solution space for measurement configurations. Performance is evaluated using all three specific criteria: minimizing the number of non-detectable scenarios, maximizing the damage detectability and minimizing the average time to detection. Multiobjective optimization using GA is carried out to identify sensor locations for measurement system that offers the best performance based on the specified criteria. Since all criteria are considered in this step, multi-objective optimization yields a set of non-dominated solutions (Pareto-optimal 
solutions). Therefore, MCDM methods (PEG-MCDM and PROMETHEE) are adopted to provide

340 decision support for selecting the best compromise solution.

\section{Case study}

342 To illustrate the performance of the approach for measurement system configuration, a railway truss

343 bridge in Zangenberg, Germany has been selected. This $80-\mathrm{m}$ steel bridge is composed of two parallel

344 trusses each having 77 members. Their properties are summarized in Table 2. The truss members

345 are made of steel having an elastic modulus of $200 \mathrm{GPa}$ and a density of $7870 \mathrm{~kg} / \mathrm{m} 3$. A finite

346 element analysis that includes traffic loading and temperature variation provides responses (strains)

347 that are taken as the measurement from continuous monitoring. Traffic loading is simulated by

348 applying a randomly generated vertical load (0-19 tonnes) at each node in the bottom chords. A load

349 of 19 tonnes is equivalent to an axle load of a railway locomotive. Daily and seasonal variations are

350 simulated as temperature loads. Temperature differences between top and bottom chords due to

351 solar radiation are also taken into account in the simulations.

352 One truss of the bridge fixed at both ends is modelled (Figure 2). Although this is not the boundary

353 conditions that were designed for the bridge, two fixed ends represent the upper-bound worst case

354 for supports that have deteriorated with age. Damage scenarios are generated where each scenario

355 represents axial-stiffness reduction of a member. Potential configurations are evaluated based on

356 the performance of detecting these damage scenarios. Two data-interpretation methods (MPCA and

357 RRA) for damage detection are adopted in this study.

358 In the first step, a global search is used to estimate the maximum performance of configurations in

359 terms of the increasing number of sensors. For every number of sensors, the maximum performance

360 is estimated by minimizing the number of non-detectable scenarios. Seventy-seven damage

361 scenarios are generated where each scenario represents $50 \%$ axial stiffness reduction of a member.

362 The results of the first step for both MPCA and RRA are shown in Figure 3. It is demonstrated that

363 MPCA can detect more scenarios than RRA. For both methods, the number of non-detectable 
damage scenarios initially reduces rapidly when the number of sensors increases. However, the reduction tapers off and the improvement of the performance becomes marginal when the number of sensors is greater than 24. For MPCA, the number of non-detectable scenarios decreases from 45 to 15 when the number of sensors is increased from 4 to 24 . Adding more sensors can only decrease the non-detectable scenario by 15 scenarios. For RRA, a reduction of 27 non-detectable scenarios is gained by adding the sensors from 4 to 24 . Increasing the number of sensors from 24 to 77 only reduced the number of non-detectable scenarios by 12 scenarios. These results show that adding more sensors will only result in small improvement of the system performance. Therefore, 24 sensors are decided for this measurement system.

Given the number of sensors to be employed, the measurement system is configured using a multiobjective optimization procedure and MCDM approaches. In the multi-objective optimization procedure, objective functions are minimizing the number of non-detectable scenarios, maximizing the damage detectability and minimizing the average time to detection. Figure 4 and 5 show the pareto-optimal solutions for both MPCA and RRA. Time to detection for RRA is much smaller than that of MPCA. On the other hand, MPCA is able to detect more damage scenarios and has higher detectability than RRA. These results indicate that RRA is able to detect damage faster than MPCA but MPCA is better in terms of damage detectability. The Pareto-optimal solutions are then ranked using PEG-MCDM and PROMETHEE. Table 3 and 4 show the ranks of the Pareto-optimal solutions for MPCA and RRA respectively. While PROMETHEE ranks the solutions based on the preference flow, $\varphi$, ranking in PEG-MCDM is based on the distance of the solution to the Pareto-compromise solution. Preference flow, $\varphi\left(S_{i}\right)$, is the absolute preference of the solution $S_{i}$ over all other solutions in the solution set. Distance represents the proximity of the solution to the Paretocompromise solution that is mutually agreeable for all objectives. For MPCA, employing the PEGMCDM procedure, the Pareto-compromise solution mutually agreeable for all objectives is a configuration with the value of criteria $1=18.5$, that of criteria $2=120.6$ and that of criteria $3=14.2$. The closest solution to this is configuration 7 as shown in Table 3. This configuration is however 
ranked as the $3^{\text {rd }}$ place when PROMETHEE is used for outranking. On the other hand, the best configuration (number 8 ) from PROMETHEE is ranked as the $2^{\text {nd }}$ place when using PEG-MCDM. For RRA, while the best-compromise configuration (number 13) from PEG-MCDM is ranked $4^{\text {th }}$ in the results when using PROMETHEE, the best-compromise configuration (number 16) from PROMETHEE is ranked in $2^{\text {nd }}$ when using PEG-MCDM. These results show that the best compromise configuration defined by using PROMETHEE and PEG-MCDM are different. This demonstrates that a compromise solution with mutually agreeable objectives is not necessarily the preferred solution using preference-based outranking strategy.

For situations where information related to the relative preference of criteria is not available or limited, it is preferable to employ PEG-MCDM method since it provides a solution that mutually satisfies all criteria. On the other hand, when preferences information is available and it is possible to build mathematical models of them, PROMETHEE is a better option. This method provides the best compromise solution based on various preference forms. Results indicate that multi-criteria decision-making methods are capable of providing support for selecting the best compromisemeasurement system.

In order to take advantages of many data-interpretation methods, engineers may decide to employ simultaneously several model-free data-interpretation methods. For such situations, a compromise solution which accommodates several methods is desirable. A solution for this can be obtained in two ways. The first is by configuring optimal measurement-system for each method and taking the union of these optimal configurations as the best compromise solution. However, this may result in excessive number of sensors. For this case study, a union of best compromise configuration for MPCA and RRA results in 38 sensors. Alternatively, a compromise solution can be obtained by treating several methods as a combined method and performing configuration based on the evaluation of the combined performance for each potential configuration. This case is referred to as optimized combination. 
Figure 6 shows respectively the optimum configurations resulting from use of MPCA, RRA, the union of these solutions and the optimized combination. Mid-span, bottom chord is one of the most common locations for sensors that is often intuitively selected by engineers. As shown in the figure, no sensor is placed in this location. This demonstrates that the methodology uncovers solutions that would not have been found using engineering judgment alone. For MPCA, no sensor is placed at bottom chord while sensors are mainly distributed at the bottom chord for RRA. The results demonstrate that different methods result in different measurement configurations. and their combinations using three criteria. The performance of the measurement systems in the sensors). As compared with MPCA, a direct combination of optimum configurations for MPCA and RRA only improves the performance in terms of time to detection. This is because such combination places additional sensors at non-informative places. On the other hand, a better performance in all three criteria is shown for the case of an optimized combination. These results demonstrate that the proposed approach is able to combine results of various model-free data-interpretation methods. Finally, engineers may uncover non-intuitive solutions using the approach described in this paper.

\section{Conclusions}

432 The following conclusions are drawn from this research.

- The proposed approach for measurement system configuration is able to accommodate model-free (non-physics-based) data interpretation methods for damage detection of continuously monitored structures. The approach is also applicable for situations where several model-free methods are used for data interpretation. The methodology may uncover solutions that would not have been found using engineering judgement alone.

- When using several data-interpretation methods for damage identification, measurement systems should be configured by optimizing simultaneously all their objective functions 
rather than using the union of best compromise measurement locations that are separately identified for each method.

- The number of non-detectable scenarios, the damage detectability and the average time to detection are useful metrics for configuring measurement systems when moving principal component analysis (MPCA) and robust regression analysis (RRA) are used for data interpretation.

- Damage scenario generation and multi-objective optimization of key metrics are helpful for measurement system configuration when data is interpreted using model-free methods.

- Multi-criteria decision-making (MCDM) methods such as Preference Ranking Organization

Future work involves a development of a model-free data-interpretation approach that combines

MPCA and RRA methods. Taking into account thermal response for improving structural identification is another current research topic.

455

456

457 METHod for Enrichment Evaluation (PROMETHEE) and Pareto-Edgeworth-MCDM (PEGMCDM) can provide support for selecting the best compromise measurement-system configuration.

\section{Acknowledgements}

This work was funded by Swiss National Science Foundation under contract no. 200020-126385. The authors would like to thank A. Nussbaumer for his contributions related to the case study.

\section{References}

Andersen, R. (2008). Modern methods for robust regression, SAGE Publications, Inc.

Aref, A. J., Alampalli, S., and He, Y. (2005). "Performance of a fiber reinforced polymer web core skew bridge superstructure. Part I: field testing and finite element simulations." Composite Structures, 69(4), 491-499.

ASCE. (2011). "Structural Identification of Constructed Facilities." Structural Identification Comittee, American Society of Civil Engineers.

Behzadian, M., Kazemzadeh, R. B., Albadvi, A., and Aghdasi, M. (2010). "PROMETHEE: A comprehensive literature review on methodologies and applications." European Journal of Operational Research, 200(1), 198-215.

Bel Hadj Ali, N., and Smith, I. F. C. (2010). "Dynamic behavior and vibration control of a tensegrity structure." International Journal of Solids and Structures, 47(9), 1285-1296. 
Brans, J.-P., and Mareschal, B. (2005a). "Promethee Methods." Multiple Criteria Decision Analysis: State of the Art Surveys, 163-186.

Brans, J. P. (1982). "L'ingénierie de la décision: élaboration d’instruments d'aide à la décision. La méthode PROMETHEE." Laide a la Decision: Nature, Instrument s et Perspectives Davenir, R. Nadeau and M. Landry, eds., Presses de l'Université Laval, Quebec. Canada, 183-214.

Brans, J. P., and Mareschal, B. (2005b). "Promethee Methods." Multiple Criteria Decision Analysis: State of the Art Surveys, 163-186.

Brans, J. P., Vincke, P., and Mareschal, B. (1986). "How to select and how to rank projects: The Promethee method." European Journal of Operational Research, 24(2), 228-238.

Cha, Y.-J., Raich, A., Barroso, L., and Agrawal, A. (2011). "Optimal placement of active control devices and sensors in frame structures using multi-objective genetic algorithms." Structural Control and Health Monitoring.

Chen, S. R., and Wu, J. (2010). "Dynamic Performance Simulation of Long-Span Bridge under Combined Loads of Stochastic Traffic and Wind." Journal of Bridge Engineering, 15(3), 219-230.

Coello Coello, C. A., Lamont, G. B., and Van Veldhuizen, D. A. (2007). Evolutionary Algorithms for Solving MultiObjective Problems, Springer US.

Fleming, P. J., Purshouse, R. C., and Lygoe, R. J. (2005). "Many-Objective Optimization: An Engineering Design Perspective." Evolutionary Multi-Criterion Optimization, 14-32.

Goulet, J.-A., Kripakaran, P., and Smith, I. F. C. (2010). "Multimodel Structural Performance Monitoring." Journal of Structural Engineering, 136(10), 1309-1318.

Grierson, D. E. (2008). "Pareto multi-criteria decision making." Advanced Engineering Informatics, 22(3), 371384.

Hou, Z., Noori, M., and St. Amand, R. (2000). "Wavelet-based approach for structural damage detection." Journal of Engineering Mechanics, 126(7), 677-683.

Hubert, M., Rousseeuw, P. J., and Branden, K. V. (2005). "ROBPCA: A new approach to robust principal component analysis." Technometrics, 47, 64-79.

Jaishi, B., and Ren, W.-X. (2006). "Damage detection by finite element model updating using modal flexibility residual." Journal of Sound and Vibration, 290(1-2), 369-387.

Jajo, N. (2005). "A Review of Robust Regression and Diagnostic Procedures in Linear Regression." Acta Mathematicae Applicatae Sinica (English Series), 21(2), 209-224.

Kang, F., Li, J.-j., and Xu, Q. (2008). "Virus coevolution partheno-genetic algorithms for optimal sensor placement." Advanced Engineering Informatics, 22(3), 362-370.

Koh, C. G., and Thanh, T. N. (2009). "Challenges and Strategies in Using Genetic Algorithms for Structural Identification." Soft Computing in Civil and Structural Engineering, B. H. V. Topping and Y. Tsompanakis, eds., Saxe-Coburg Publications, Stirlingshire, UK, 203-226.

Kripakaran, P., and Smith, I. F. C. (2009). "Configuring and enhancing measurement systems for damage identification." Advanced Engineering Informatics, 23(4), 424-432.

Lanata, F., and Grosso, A. D. (2006). "Damage detection and localization for continuous static monitoring of structures using a proper orthogonal decomposition of signals." Smart Materials and Structures, 15(6), 1811-1829.

Li, D. S., Li, H. N., and Fritzen, C. P. (2007). "The connection between effective independence and modal kinetic energy methods for sensor placement." Journal of Sound and Vibration, 305(4-5), 945-955.

Li, Z. N., Tang, J., and Li, Q. S. (2004). "Optimal sensor locations for structural vibration measurements." Applied Acoustics, 65(8), 807-818.

Liu, M., Frangopol, D. M., and Kim, S. (2009). "Bridge Safety Evaluation Based on Monitored Live Load Effects." Journal of Bridge Engineering, 14(4), 257-269.

Liu, W., Gao, W.-c., Sun, Y., and Xu, M.-j. (2008). "Optimal sensor placement for spatial lattice structure based on genetic algorithms." Journal of Sound and Vibration, 317(1-2), 175-189.

Meo, M., and Zumpano, G. (2005). "On the optimal sensor placement techniques for a bridge structure." Engineering Structures, 27(10), 1488-1497. 
Ni, Y. Q., Zhou, H. F., Chan, K. C., and Ko, J. M. (2008). "Modal Flexibility Analysis of Cable-Stayed Ting Kau Bridge for Damage Identification." Computer-Aided Civil and Infrastructure Engineering, 23(3), 223-236.

Omenzetter, P., and Brownjohn, J. M. W. (2006). "Application of time series analysis for bridge monitoring." Smart Materials and Structures, 15(1), 129-138.

Papadimitriou, C. (2004). "Optimal sensor placement methodology for parametric identification of structural systems." Journal of Sound and Vibration, 278(4-5), 923-947.

Posenato, D., Kripakaran, P., Inaudi, D., and Smith, I. F. C. (2010). "Methodologies for model-free data interpretation of civil engineering structures." Computers \& Structures, 88(7-8), 467-482.

Posenato, D., Lanata, F., Inaudi, D., and Smith, I. F. C. (2008). "Model-free data interpretation for continuous monitoring of complex structures." Advanced Engineering Informatics, 22(1), 135-144.

Rakesh, K. K., and et al. (2008). "Placement Optimization of Distributed-Sensing Fiber-Optic Sensors Using Genetic Algorithms." AIAA Journal, 46(4), 824.

Rao, A. R. M., and Ganesh, A. (2007). "Optimal placement of sensors for structural system identification and health monitoring using a hybrid swarm intelligence technique." Smart Materials and Structures, 16(6), 2658.

Raphael, B., and Smith, I. F. C. (2003). "A direct stochastic algorithm for global search." Applied Mathematics and Computation, 146(2-3), 729-758.

Robert-Nicoud, Y., Raphael, B., Burdet, O., and Smith, I. F. C. (2005a). "Model Identification of Bridges Using Measurement Data." Computer-Aided Civil and Infrastructure Engineering, 20(2), 118-131.

Robert-Nicoud, Y., Raphael, B., and Smith, I. F. C. (2005b). "Configuration of measurement systems using Shannon's entropy function." Computers and structures, 83(8-9), 599-612.

Saitta, S., Raphael, B., and Smith, I. F. C. (2005). "Data mining techniques for improving the reliability of system identification." Advanced Engineering Informatics, 19(4), 289-298.

Saitta, S., Raphael, B., and Smith, I. F. C. (2006). "Rational Design of Measurement Systems using Information Science." IABSE Symposium Report, 92(25), 37-44.

Sastry, K. (2007). "Single and Multiobjective Genetic Algorithm Toolbox for Matlab in C++ (IlligAL Report No. 2007017)." IL: University of Illinois at Urbana-Champaign, Urbana, USA.

Tongpadungrod, P., Rhys, T. D. L., and Brett, P. N. (2003). "An approach to optimise the critical sensor locations in one-dimensional novel distributive tactile surface to maximise performance." Sensors and Actuators A: Physical, 105(1), 47-54.

Wang, H., Song, Z., and Wang, H. (2002). "Statistical process monitoring using improved PCA with optimized sensor locations." Journal of Process Control, 12(6), 735-744.

Xu, J., Johnson, M. P., Fischbeck, P. S., Small, M. J., and VanBriesen, J. M. (2010). "Robust placement of sensors in dynamic water distribution systems." European Journal of Operational Research, 202(3), 707-716.

Yan, A. M., Kerschen, G., De Boe, P., and Golinval, J. C. (2005). "Structural damage diagnosis under varying environmental conditions--part II: local PCA for non-linear cases." Mechanical Systems and Signal Processing, 19(4), 865-880. 
Click here to download Table: Ms.No. BEENG-696 - Tables.doc

Table 1. Estimated execution times for single and multi-objective optimization

\begin{tabular}{lll}
\hline Search algorithm & Number of evaluations & Estimated execution time \\
\hline Single objective & 400 & 0.6 hour \\
Three objectives & 40000 & 60 hours \\
Five objectives & 4000000 & 6000 hours \\
\hline
\end{tabular}

Table 2. Properties of truss members of a railway bridge in Zangenberg, Germany

\begin{tabular}{lcccc}
\hline Member type & Area $\left(\mathbf{m}^{2}\right)$ & $\mathrm{I}_{\mathbf{x}}\left(\mathrm{m}^{4}\right)$ & $\mathrm{I}_{\mathrm{y}}\left(\mathrm{m}^{4}\right)$ & Length $(\mathrm{m})$ \\
\hline Top chord & $5.15 \times 10^{-2}$ & $2.27 \times 10^{-3}$ & $2.58 \times 10^{-3}$ & 4.00 \\
Bottom chord & $3.03 \times 10^{-1}$ & $1.47 \times 10^{-3}$ & $1.46 \times 10^{-3}$ & 2.00 \\
Vertical & $2.19 \times 10^{-2}$ & $1.21 \times 10^{-3}$ & $4.24 \times 10^{-5}$ & 4.00 \\
Diagonal & $3.69 \times 10^{-2}$ & $9.70 \times 10^{-4}$ & $4.16 \times 10^{-3}$ & 5.66 \\
Small diagonal & $2.19 \times 10^{-2}$ & $1.21 \times 10^{-3}$ & $4.24 \times 10^{-5}$ & 5.66 \\
\hline
\end{tabular}

Table 3. Outranking solutions of the Pareto-optimum set for MPCA

\begin{tabular}{lcccc}
\hline Rank & $\varphi$ & $\begin{array}{c}\text { PROMETHEE } \\
\text { (Configuration number) }\end{array}$ & Distance & $\begin{array}{c}\text { PEG-MCDM } \\
\text { (Configuration number) }\end{array}$ \\
\hline 1 & 9.3 & 8 & $4.8 \mathrm{E}-03$ & 7 \\
2 & 9.1 & 13 & $6.1 \mathrm{E}-03$ & 8 \\
3 & 8.5 & 7 & $1.0 \mathrm{E}-02$ & 4 \\
4 & 7.4 & 5 & $1.1 \mathrm{E}-02$ & 5 \\
5 & 6.9 & 4 & $1.8 \mathrm{E}-02$ & 11 \\
\hline
\end{tabular}

Table 4. Outranking solutions of the Pareto-optimum set for RRA

\begin{tabular}{lcccc}
\hline Rank & $\varphi$ & $\begin{array}{c}\text { PROMETHEE } \\
\text { (Configuration number) }\end{array}$ & Distance & $\begin{array}{c}\text { PEG-MCDM } \\
\text { (Configuration number) }\end{array}$ \\
\hline 1 & 9.8 & 16 & $9.9 \mathrm{E}-03$ & 13 \\
2 & 5.1 & 19 & $1.1 \mathrm{E}-02$ & 16 \\
3 & 3.4 & 18 & $1.5 \mathrm{E}-02$ & 9 \\
4 & 3.0 & 13 & $1.6 \mathrm{E}-02$ & 12 \\
5 & 1.9 & 4 & $1.7 \mathrm{E}-01$ & 18 \\
\hline
\end{tabular}

Table 5. Performance of the optimum configuration for different data-interpretation methods. Criteria 1 is the number of non-detectable damage scenarios; Criteria $\mathbf{2}$ is the damage detectability (\%); and Criteria $\mathbf{3}$ is the average time to detection (days).

\begin{tabular}{lcccc}
\hline Data-interpretation method & Number of sensors & Criteria 1 & Criteria 2 & Criteria 3 \\
\hline MPCA & 24 & 17 & 86.2 & 139.5 \\
RRA & 24 & 42 & 45.0 & 3.0 \\
Union of MPCA and RRA & 38 & 17 & 86.3 & 47.4 \\
Optimized combination of MPCA and RRA & 24 & 13 & 92.5 & 87.6 \\
\hline
\end{tabular}



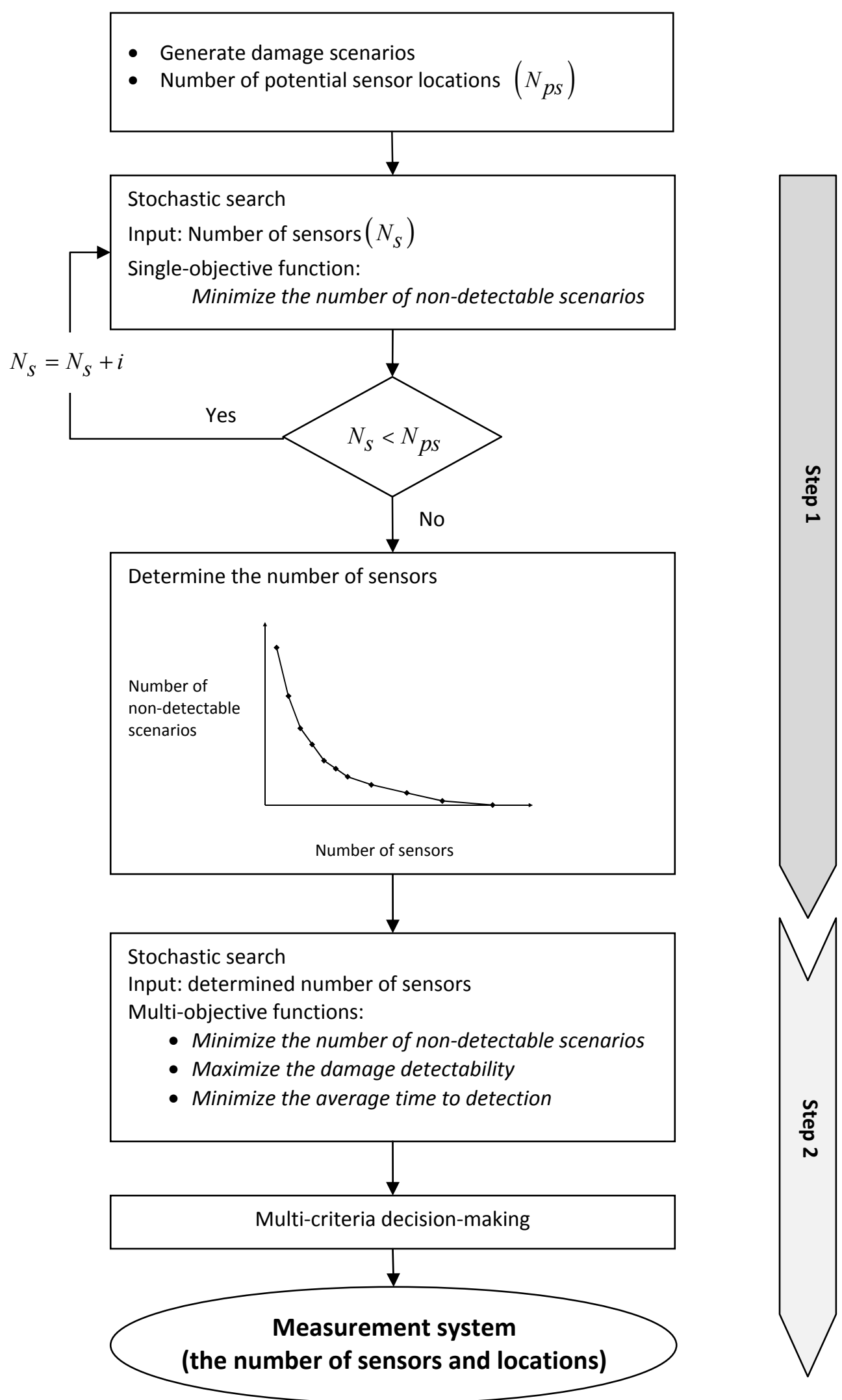

Figure 1. A flowchart of a systematic approach for measurement-system configuration 
Click here to download Figure: Fig2.pdf

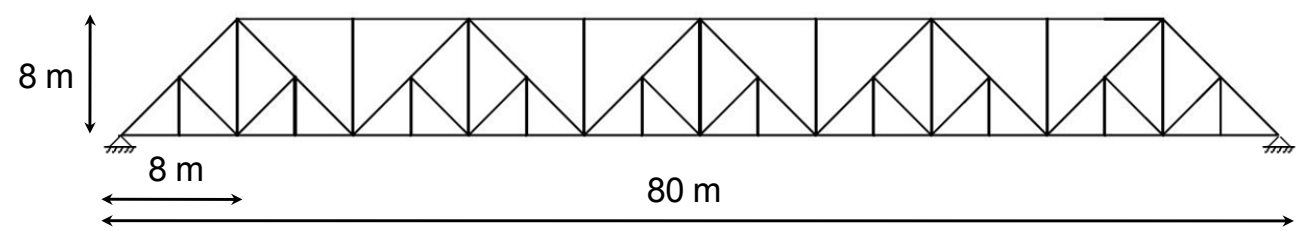

Figure 2. A truss structure inspired by a 80-m railway bridge 


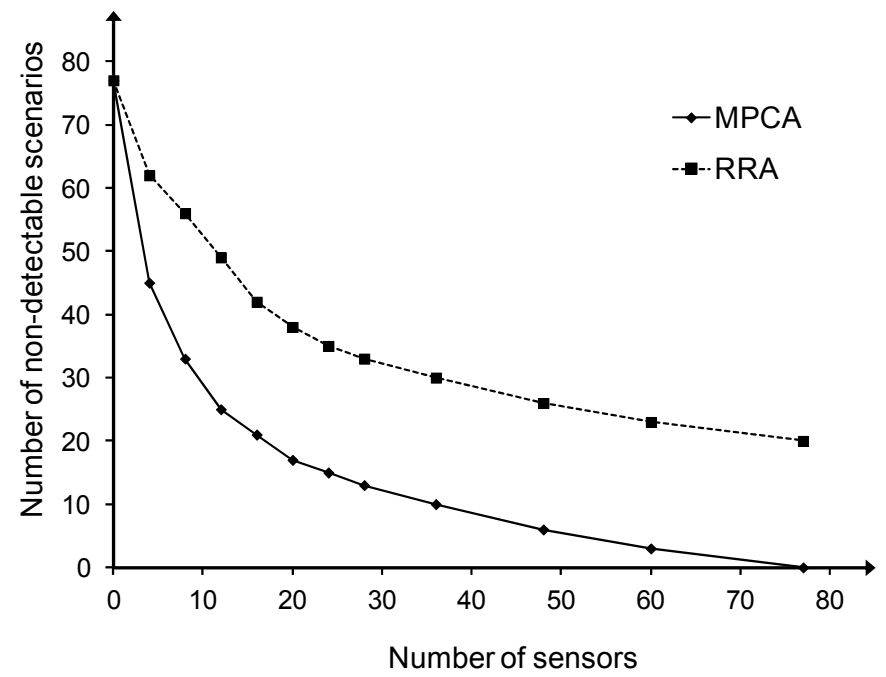

Figure 3. Number of non-detectable scenarios corresponding to the number of sensors 

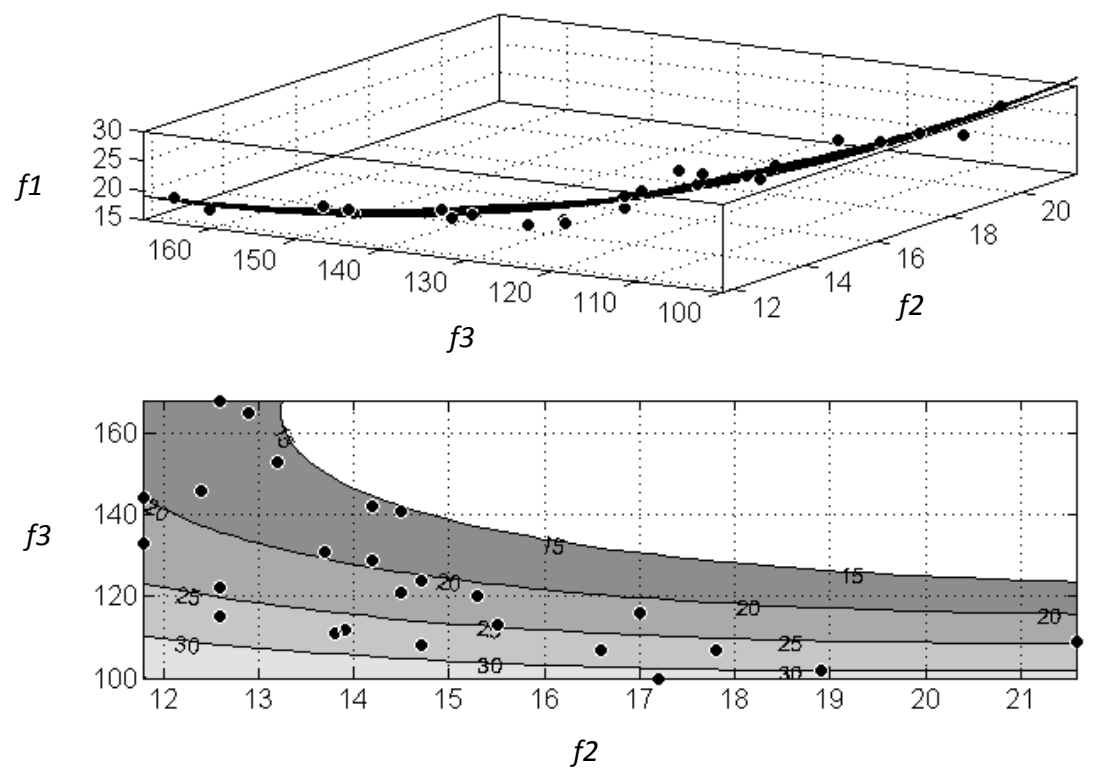

Figure 4. Pareto-optimal solutions for MPCA. $f 1$ is the number of non-detectable damage scenarios; $f 2$ is the average minimum detectable damage-level (\%); and $f 3$ is the average time to detection (days). The contour values in the lower figure refer to values of $f 1$. 

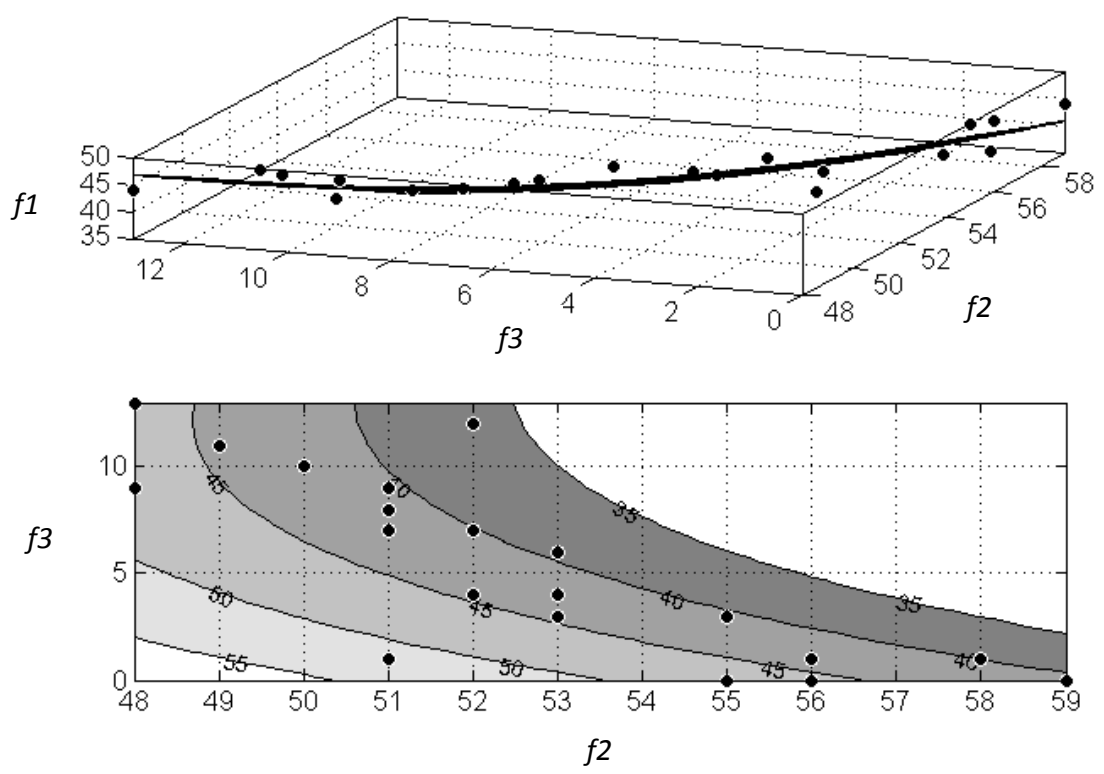

Figure 5 Pareto-optimal solutions for RRA. $f 1$ is the number of non-detectable damage scenarios; $f 2$ is the average minimum detectable damage-level (\%); and $f 3$ is the average time to detection (days). The contour values in the lower figure refer to values of $f 1$. 


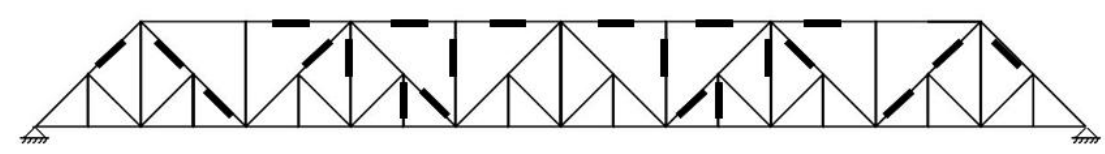

a) Moving principal component analysis (MPCA)

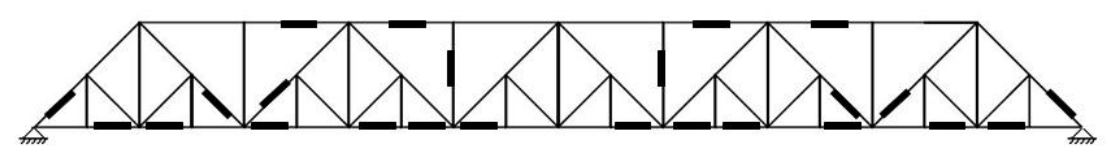

b) Robust regression analysis (RRA)

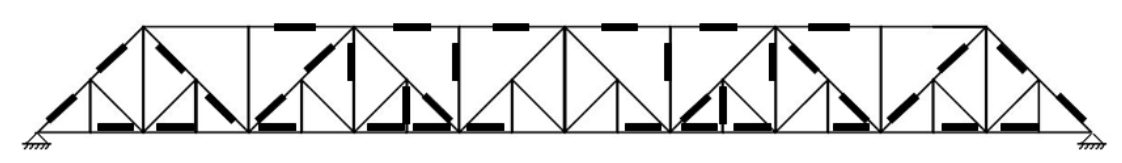

c) Union of configurations (a) and (b)

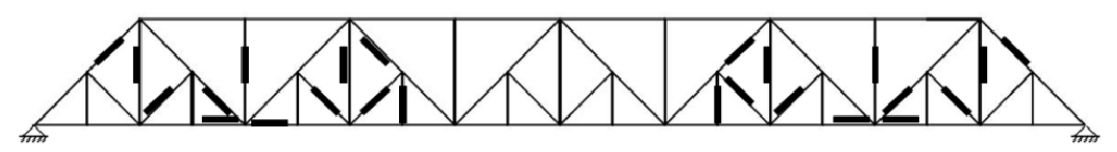

d) Optimized combination of MPCA and RRA

Figure 6. Measurement system configuration for situations where (a) MPCA, (b) RRA, (c) union of a and b; and (d) optimized combination of MPCA and RRA are employed for data interpretation. 